\title{
Germanica
}

\section{Non, ce pays n'est pas pour la vieille femme. Vieillesse et féminité dans La Pianiste d'Elfriede Jelinek}

No Country for Old Women. Aging and Femininity in Elfriede Jelinek's Novel

ThePiano Teacher.

Kein Land für alte Frauen. Alter und Weiblichkeit im Elfriede Jelineks Roman

Die Klavierspielerin

\section{Patrick Bergeron}

\section{OpenEdition}

Journals

Édition électronique

URL : http://journals.openedition.org/germanica/1558

DOI : 10.4000/germanica. 1558

ISSN : 2107-0784

Éditeur

Université de Lille

Édition imprimée

Date de publication : 10 juillet 2012

Pagination : 95-108

ISBN : 9782913857292

ISSN : 0984-2632

Référence électronique

Patrick Bergeron, « Non, ce pays n'est pas pour la vieille femme. Vieillesse et féminité dans La Pianiste d'Elfriede Jelinek », Germanica [En ligne], 50 | 2012, mis en ligne le 19 juillet 2012, consulté le 06 octobre 2020. URL : http://journals.openedition.org/germanica/1558 ; DOI : https://doi.org/10.4000/ germanica.1558

Ce document a été généré automatiquement le 6 octobre 2020.

(c) Tous droits réservés 


\title{
Non, ce pays n'est pas pour la vieille femme. Vieillesse et féminité dans La Pianiste d'Elfriede Jelinek
}

\author{
No Country for Old Women. Aging and Femininity in Elfriede Jelinek's Novel \\ ThePiano Teacher. \\ Kein Land für alte Frauen. Alter und Weiblichkeit im Elfriede Jelineks Roman \\ Die Klavierspielerin
}

Patrick Bergeron

\section{«[...] und los gehts mit der Alterung!» Elfriede Jelinek ${ }^{1}$}

L'idée ne nous viendrait pas de lire une œuvre d'Elfriede Jelinek dans l'espoir d'y trouver une vision idyllique de l'Autriche ou une évocation harmonieuse des rapports homme-femme. L'Autrichienne s'est acquise une réputation de Nestbeschmutzerin ${ }^{2}$ à force de pourfendre les spectres de l'austro-fascisme ou de critiquer ses compatriotes, ces «Barbares repus dans un pays où la barbarie règne culturellement parlant ${ }^{3}$. » Sur le plan sentimental, elle ne donne pas non plus dans la dentelle. Pour elle, la violence sexuelle exercée contre les femmes (avec leur complicité) fait intrinsèquement partie de notre civilisation capitaliste et patriarcale.

En fait, Jelinek a élaboré une écriture nourrie de négativité. « Je ne fonctionne que dans la mesure où je suis capable de critiquer ce qui existe ${ }^{4}$ ", confiait-elle en entrevue à Hans-Jürgen Greif. Nancy Huston perçoit dans cette position négative le summum du désespoir: "Impossible de caractériser l'œuvre d'Elfriede Jelinek autrement que comme une œuvre de haine. [...] Le ton omniprésent, invariable, est celui de l'ironie dévastatrice ${ }^{5}$. N Non seulement les textes de Jelinek s'appliquent-ils à "démontrer l'impuissance des femmes ", poursuit Huston, mais ils

enfoncent le même clou: l'homme est la norme, la femme est l'Autre, l'homme occupe toute la place, il dirige, crée et transforme le monde, et la femme ne peut que regarder ce monde de l'extérieur, tel un extraterrestre ou un enfant. Jelinek 
prive les femmes (sauf elle-même) de toute individualité, toute forme de puissance, toute initiative et toute liberté6.

Nul ne sera surpris, dès lors, de ne pas retrouver chez Jelinek d' « éloge de la vieillesse ", pour reprendre le titre d'un livre d'Hermann Hesse. Elle donne en effet une image plutôt rébarbative du vieillissement, particulièrement en ce qui concerne le vieillir féminin, car l'âge, synonyme pour elle de disgrâce, de détérioration et de putrescence, tend à accroître l'impuissance des femmes.

On le voit clairement dans Lust (1989). C'est avant tout par sa beauté physique que Gerti peut espérer plaire à Michael, son jeune amant. En même temps, elle sait que l'âge a pour effet de flétrir ses charmes. La vision de belles jeunes filles blondes, au centre de ski, n'offre donc rien de réconfortant:

Et ces chères têtes blondes, bouclées, permanentées, se prendront aux sangles de nos regards envieux, mesdames, vous dont le cheveu rare n'émeut plus personne, mais qui vous laissez émouvoir par des feuilletons télévisés. [...] Soyons sincères, nous leur envions leurs visages multiples, tandis que l'âge nous rend de plus en plus semblables à nous-mêmes, et la plus coûteuse des eaux de toilette ne fait pas de nous des femmes de la plus belle eau?

Gerti vit une situation semblable à celle d'Erika Kohut dans La Pianiste : celle d'« une femme qui, amoindrie par l'âge, ne peut que redouter la concurrence de ses cadettes ${ }^{8}$." Avidité (2000) s'appuie sur une expérience analogue. Jelinek y dénonce l'injustice qui contraint la femme à se valoriser par l'entremise de son corps et la met à l'écart aussitôt qu'apparaissent les premières traces du vieillissement. L'homme a le beau jeu, car même en prenant de l'âge, il peut refaire sa vie et s'attirer les faveurs de femmes plus jeunes.

L'étude qui suit s'appuie principalement sur La Pianiste (1983). Ce septième roman de Jelinek est sans doute son œuvre la plus connue, en partie grâce au film qu'en a tiré Michael Haneke en 2001. C'est peut-être aussi l'œuvre dans laquelle Jelinek propose l'évocation la plus inquiétante du vieillissement chez les femmes. Le récit est construit autour de deux figures féminines. La première, Erika Kohut, est une fausse vieille (elle n'a que 36 ans), qui sent cruellement peser sur elle les conventions sociales en matière de coquetterie, de féminité et d'apparence jeune. Professeur de piano à la personnalité sadomasochiste, elle vit confinée dans une cellule (donc une prison) familiale matrilinéaire, où l'autre figure féminine d'importance, sa mère âgée, l'épie et l'étouffe constamment par sa nature possessive et paranoïaque. J'aimerais montrer que cette représentation de la mère et de la fille sous l'angle de leur âge avancé est conforme à l'esprit dans lequel Jelinek conçoit son travail d'écrivain : l'écriture permet de moduler une critique sociale et de révéler les rouages des servitudes modernes. Les femmes, nous dit Jelinek, ne cessent de faire les frais d'un système culturel basé sur le culte de l'image, les rapports de force et le profit. Le vieillissement, à toutes fins pratiques, est l'ennemi juré de leur beauté.

\section{Ô vieillesse ennemie !}

Les propos du Prince dans La Belle au Bois Dormant (2003) corroborent cet antagonisme vieillesse/beauté : «Et c'est parti pour le vieillissement! Dans cent ans, il n'y aura plus de baisers, mais des liftings en masse! Ici, nous ne voulons évidemment pas voir le temps comme adversaire de l'éternité, mais tout au plus comme adversaire de la beauté féminine ${ }^{9} .$. » Par-delà le sarcasme inhérent à la tirade princière, Jelinek 
montre bien son ancrage dans le plus contemporain des contextes. Depuis Wir sind lockvögel baby! (1970) jusqu'à Winterreise (2011), l'Autrichienne a élaboré son œuvre à une époque durant laquelle nos rapports à la vieillesse ont rapidement (et substantiellement) changé.

La perspective d'être vieux reste une idée déprimante. Elle se nourrit, d'un point de vue social, du stéréotype assimilant les aînés à des individus amoindris et dépendants, qui constituent, par les soins de santé et les régimes de pension dont ils bénéficient, un fardeau de plus en plus lourd à porter par une population active qui, pour sa part, va décroissant. Sur un plan individuel, la perception négative de la vieillesse s'enracine dans l'appréhension de la ruine du corps. Qui l'a mieux résumée que Norman Thayer (Henry Fonda), le professeur à la retraite dans le film de Mark Rydell, On Golden Pond (1981)? Alors que sa femme Ethel (Katharine Hepburn) écoule ses vieux jours avec sérénité et entrain (comme quoi ce n'est pas impossible !), il clame quant à lui avec humeur: "Do you think it's funny being old? My whole goddam body's falling apart ${ }^{10}$.» Il devient en effet difficile de détourner sa pensée quand l'organisme se délabre.

Outre les forces déclinantes, deux autres perceptions confortent cette stéréotypie négative du vieil âge : la soi-disant inutilité sociale du vieillard et sa proximité avec la mort. On se souviendra du jeune homme, chez La Fontaine, qui s'étonnait de voir un octogénaire occupé à planter :

À quoi bon charger votre vie

Des soins d'un avenir qui n'est pas fait pour vous ${ }^{11}$ ?

Être vieux, estime le naï jouvenceau, signifie ne plus appartenir au temps présent. Jonathan Swift enfonce le clou, comme dirait Nancy Huston. Rappelons-nous de ses Struldbruggs, ce peuple d'immortels que croise Gulliver au royaume de Luggnagg. Le héros ne tarde pas à comprendre que leur immortalité a tout d'une malédiction, puisque si les Struldbruggs ne meurent pas, leurs corps continuent néanmoins d'accumuler les marques de la décrépitude. Par conséquent, ces êtres présentent, au physique, l'aspect de vieillards hideusement défigurés par le temps: "Leur vue était révoltante. Les femmes étaient encore plus horribles que les hommes. Toutes joignaient aux difformités ordinaires à l'extrême vieillesse un certain air de spectre plus marqué, suivant le nombre de leurs années, et dont l'effet ne peut se décrire ${ }^{12}$. " L'ironie sexiste ne nous aura pas échappé : les femmes vieillissent encore plus mal que les hommes... Il eût fallu, pour y faire contrepoids, « des liftings en masse », comme le disait le Prince dans La Belle au Bois Dormant.

Le topos de la laideur des vieilles femmes s'appuie sur une longue tradition dans l'imaginaire. On peut en suivre le fil d'Hans Baldung (Les Trois Âges de la vie [1510] et Les Sept Âges de la vie [1544]) à Francisco Goya (Linda maestra! [1797]) et Gustav Klimt (Les Trois Âges de la femme [1905]). Alors que le vieillard suscite traditionnellement l'image du bon patriarche (Abraham, Moïse, saint Nicolas / le Père Noël...), on chercherait longtemps un archétype de «bonne matriarche ». La vieille femme éveille plutôt l'image de la sorcière. Dans les contes traditionnels par exemple, pour une gentille grand-mère comme celle du Petit Chaperon Rouge, on trouve une myriade de vieilles marâtres, reines ou fées malveillantes. De même, la comparaison entre des jeunes filles et des femmes âgées se fait rarement au profit de ces dernières, dans la mesure où « rien ne corrige la différence irréductible Vieille/ Jeune $^{13}$.» En peignant candidement son corps d'octogénaire nue dans Autoportrait 
(1980), Alice Neel défiait ainsi un vieux consensus assimilant le corps des femmes âgées à un objet répugnant ou honteux. Nous sommes loin, chez Neel, de l'entrée en décomposition à laquelle Jelinek assimile le vieillir féminin.

$\mathrm{Au}$ fond, Jelinek, particulièrement dans La Pianiste, n'a pas l'intention de réconcilier la femme avec « la prison de son corps vieillissant ${ }^{14}$ ». Non seulement le temps induit-il un délabrement qui a pour effet d'enlaidir les femmes (Erika Kohut, une mi-trentenaire, en fait déjà l'expérience ${ }^{15}$ ), mais celles-ci sont les esclaves d'un jeunisme les laissant croire qu'elles peuvent préserver une apparence jeune en recourant à une gamme sophistiquée de produits anti-âge. "Qu'on en finisse avec ces atours, ces niaiseries foncièrement enlaidissantes ${ }^{16}$ !», clame Jelinek avec son tranchant habituel. Nulle ne peut espérer sortir gagnante de cette lutte irraisonnée contre la nature, qui « est sale où qu'on entre en contact avec elle ${ }^{17}$ ».

Née en 1946, Jelinek fait partie de la génération qui a atteint la soixantaine dans les années 2000. Cette génération, dite «des baby-boomers » en Amérique du Nord, a déjà commencé à substituer à l'image traditionnellement négative du vieillissement une nouvelle perception du devenir vieux, positive et dynamique. Avec les boomers, l'âge de la retraite devient (si la santé n'a pas fait défection) un appendice doré après une vie professionnelle aux multiples contraintes; être retraité signifie entrer dans une période de liberté et de loisir pouvant recouvrir une durée plus longue que celle de la carrière, puisque l'espérance de vie s'est considérablement accrue depuis l'après-guerre dans les pays industrialisés. Pour David Cravitt, la façon dont les boomers assument la vieillesse ne provoque rien de moins qu'un phénomène de « dévieillissement" (de-aging): "What the Boomers are doing is, quite simply, destroying our entire concept of aging. The Boomers are, in effect, de-aging ${ }^{18}$. » Dans Méfions-nous de la nature sauvage (1985), Mme Aichholzer est l'une de ces nouvelles vieilles : «La vieille femme veut sentir des êtres grandir sous ses doigts, des hommes jeunes, même sauvages. Une jardinière de l'amour. De soixante-dix ans passés ${ }^{19}$ ! Ailleurs, Jelinek indique à son sujet qu'elle " est vieille, cette femme, et néanmoins palpite de convoitise : elle veut une existence savoureuse ${ }^{20}$. »

Tel est le défi auquel s'est attaquée la génération dont fait partie Jelinek : faire en sorte de mener " une existence savoureuse » tout en étant vieux. La culture populaire, voire de masse ${ }^{21}$, dans laquelle Jelinek puise une part considérable de son inspiration (elle ne cesse, par exemple, de faire référence aux feuilletons télévisés), est imprégnée d'un jeunisme qui repousse résolument les limites du vieil âge. " La jeunesse dont la mode fait rayonner les corps entoure les édifices ${ }^{22}$ ", écrit-elle. En ce tournant du xxie siècle, les mentalités évoluent ainsi en vue d'une acceptation plus sereine des troisième et quatrième âges. La campagne Pro-Age lancée en 2007 par la compagnie Dove le montre bien. L'entreprise a produit une série de capsules publicitaires montrant des quinquagénaires et des sexagénaires posant nues sous l'œil de la caméra, accompagnées du slogan: Beauty has no age limit. Nous traversons donc une phase dont Jelinek, fidèle à elle-même, ne va surtout retenir que l'envers négatif : c'est l'ère du Botox, du lifting et des soins anti-âge. «Aujourd'hui tout ce qui est beau est ruiné en un temps record ${ }^{23}$ ", affirme-t-elle. La femme de notre époque paraît plus que jamais dépersonnalisée et victime de la suprématie de l'image et des discours de domination sexuelle masculins. Il ne faut tout de même pas oublier de se «méfier de la nature sauvage » :

Cette nature sauvage en trompe l'œil est censée nous propulser hors de la fadeur de notre existence. Nous nous approprions la nature, la transformons à notre image 
afin qu'elle nous ressemble. Faisons entrer chez nous la fraîcheur, en flacons. Le

voici le secret de notre beauté : la fraîcheur longue durée ${ }^{24}$.

Nous étions prévenus : on ne lit guère les œuvres de Jelinek pour se réconcilier avec nos semblables. Jelinek juge d'un œil sévère « l'existence moderne » dont les « chemins [...] ne mènent nulle part ${ }^{25}$ ».

\title{
Le désespoir des vieilles
}

\author{
«... puis la porte s'ouvre sur le morne pays, le \\ mortel pays de l'amour maternel. » \\ Elfriede Jelinek ${ }^{26}$.
}

On pourrait être tentés de croire, en lisant La Pianiste, que Jelinek n'y considère que la situation, sordide à souhait, d'une trentenaire névrosée et de sa mère étouffante. La romancière semble vouloir elle-même nous persuader de la singularité du couple ErikaMme Kohut, puisqu'elle estime avoir créé un précédent: "c'est la seule fois dans la littérature, autant que je sache, où la fille séduit la mère dans une scène homosexuelle ${ }^{27}$. " Une grande partie des perversions que relate La Pianiste s'applique en effet à deux drôles d'oiseaux. Le malaise existentiel et la fêlure que dénotent les fantasmes sexuels d'Erika (consignés dans sa lettre à Walter), en même temps que les dévoiements émanant de «l'amour maternel » (espionnage, volonté de contrôle, jalousie, paranoïa...), s'expliquent dans une large mesure par la nature et le vécu atypiques de la fille et de sa mère. Pourtant, par-delà les deux Kohut, ce sont une dimension collective d'une part (Vienne, l'Autriche) et une époque précise d'autre part (la nôtre) que la romancière a placées dans son collimateur.

Jelinek nous en prévient dès l'ouverture du récit : «Vienne, ville de la musique! Dans cette ville, seul ce qui jusqu'ici a su s'imposer s'imposera encore ${ }^{28}$." Elle nous le rappelle aussi lors du dénouement :

Derrière les grilles du Burggarten de jeunes mères attaquent leur marche journalière. Les premiers interdits pleuvent sur le gravillon des allées. Du haut de leur taille les mères distillent leur fiel. [...] Dans la pâtisserie Aïda, des mères déplorent l'activité sexuelle de leurs filles qui, dès le début, leur paraît dangereusement précoce. Elles louent leurs fils qui investissent dans les études et dans le sport ${ }^{29}$.

Du fiel pour les filles, des louanges pour les fils; tout est dit. Même les mères perpétuent un héritage phallocratique. Je l'ai évoqué précédemment en rapportant les propos qu'a tenus Nancy Huston sur l'auteure de La Pianiste: ce qu'il y a de troublant chez Jelinek, c'est de voir les femmes consentir aux pressions et oppressions auxquelles le patriarcat les expose. Quiconque sait lire entre les lignes (ce qu'il ne faut cesser de faire lorsqu'on s'attaque à un texte de Jelinek) aura compris que l'Autrichienne n'estime ni normal, ni tolérable cet état de fait. Elle y voit plutôt un esclavage de plus à dénoncer et à neutraliser par le truchement du sarcasme et de l'outrance. C'est sa manière de nous convier à décrypter le récit de La Pianiste audelà des cas d'espèce que représentent le trio Érika (= la fille soumise), Mme Kohut (= la mère possessive) et Klemmer (= le jeune amant sportif, kayakiste dans le roman et hockeyeur dans le film). Comme pour les autres écrits de Jelinek, La Pianiste repose sur un dispositif narratorial compliqué, qui autorise divers degrés de lecture. La critique du vieillissement féminin, ou plutôt de la réponse sociale au vieillissement des femmes, est de ceux-là. 
Pour formuler sa critique, la romancière emprunte deux biais. La figure d'Erika lui permet tout d'abord d'évoquer le rapport névrotique à la coquetterie et à la sexualité. Le personnage de Mme Kohut lui sert ensuite à tracer le portrait d'une véritable ogresse, c'est-à-dire d'une figuration monstrueuse qui se repaît à dépecer de la chair fraîche, de la chair d'enfant - de son enfant. Mme Kohut a tout dévoré en Erika : la féminité, l'indépendance, la jeunesse, la joie de vivre, ne laissant que le «cadavre informe [d'un] professeur de piano ${ }^{30} »$.

Pour Erika, les dés sont jetés dès la naissance. Son prénom floral : «Erika, fleur de bruyère. C'est de cette fleur que cette femme tient son nom ${ }^{31}$ ", la prédestine à subir le même sort que la fleur, c'est-à-dire à vite se faner. À 36 ans, "sa propre jeunesse est passée $^{32}$ ». La pianiste se vautre "sous la couche terne, calleuse de son âge ${ }^{33}$ ». La roue du temps agit sur elle tel un rouleau compresseur : « En outre cette femme-là vieillit à vue d'œil $[. . .]^{34}$ »; «Qu'Erika sache bien que chaque année qui pour Klemmer compte encore simple, compte au moins triple à son âge ${ }^{35} »$. Souffre-t-elle d'une défaillance particulière, à l'instar de Benjamin Button, l'enfant né vieillard dans la nouvelle de Scott G. Fitzgerald ${ }^{36}$ ? Hélas non, peut-on dire, puisque le héros fitzgéraldien était tout de même dédommagé de sa décrépitude congénitale par un vieillissement inversé : il rajeunissait au fur et à mesure où il prenait de l'âge. Erika Kohut n'a pas cette chance :

Le visage d'Erika est déjà marqué par sa décomposition future. La peau de son visage se ride, les paupières se gondolent légèrement telle une feuille de papier sous l'effet de la chaleur, le tissu délicat sous les yeux se fronce en ombres bleuâtres. Audessus de la racine du nez, deux plis nets, à jamais ineffaçables. Le visage devient une enveloppe trop large, et ce processus durera encore des années, jusqu'à ce que la chair se ratatine sous la peau, disparaisse, et que la peau adhère étroitement à la tête de mort qui ne la réchauffera plus ${ }^{37}$.

Avec pareil portrait, Erika pourrait passer incognito parmi les Struldbruggs de Swift. Pourtant, à bien lire le texte de Jelinek, on comprend que cette prise d'âge accéléré permet à la romancière de s'attaquer à des mythes sociaux. Dans cette Autriche préservatrice de traditions (et de mensonges), dans cette ère contemporaine vouée à "tout ce qui est au service du patriarcat: le pouvoir, l'argent, le langage, le $\operatorname{sex}^{38}$ ", certains irritants sociaux restent indiscutés. Jelinek «[met] à nu la violence des structures patriarcales qui sont à la base des relations sociales ${ }^{39}$ ", en vertu desquelles les femmes se voient dénier une indépendance qui leur soit propre. Cette situation s'envenime lorsqu'elles vieillissent. Voilà le désolant constat que la figure d'Erika Kohut invite à dresser. Non, ce pays n'est pas fait pour les vieilles femmes.

Le mal-être que transporte Erika peut sembler résulter d'une opposition problématique entre l'art et la vie, un conflit que Tonio Kröger, le héros de Thomas Mann dans le récit éponyme, avait solutionné en optant pour la réclusion volontaire dans les hautes sphères de l'art. Erika, éminent professeur de piano, a elle aussi pris refuge dans l'art d'une manière à interpréter comme un rejet des trivialités de la vie commune. Son penchant plus affirmé pour Schubert (qui ne s'explique pas seulement par l'amour de sa musique, car Erika retient aussi le physique ingrat du compositeur et sa mort survenue tôt) suggère la présence d'une empathie identificatoire. Erika vit, dans l'art, retranchée hermétiquement de son prochain, en particulier des gens de son âge. "Plutôt le sommet de l'art que les bas-fonds du sexe ${ }^{40}$ ", stipule la loi de la mère. Or, toutes grisantes et supérieures que soient les voluptés de l'art, la musique n'apparaît jamais pour Erika comme un exutoire satisfaisant. Bien au contraire : l'art fait partie de ce qui lui a fait développer sa monomanie du vieillissement. 
Le rapport de la pianiste à la musique est l'aboutissement d'une éducation qui s'est exercée par la contrainte et qui équivaut, en ce sens, à un apprentissage négatif de la réalité. Erika maîtrise le piano parce que Mme Kohut l'y a forcée, assumant davantage un rôle de geôlière que de mère, elle qui ne cessait d'épier et de contrôler les faits et gestes de la prisonnière. Mme Kohut remplit ce rôle depuis qu'elle a accouché d'Erika : "Aussi dès qu'elle vit la motte de glaise qui venait de jaillir de son corps, elle se mit sur-le-champ à la retailler sans égards, pour l'épurer et l'affiner ${ }^{41}$. » À force de vouloir soustraire Erika des "intrigues» et autres «débauches " perpétrées par les jeunes gens, Mme Kohut a encagé sa fille dans un déséquilibre aux conséquences peu banales. Le respecté professeur fréquente des peep-shows où elle renifle des mouchoirs enduits du sperme d'inconnus. La digne bourgeoise chez qui «tout ce qui peut se fermer est fermé ${ }^{42}$ » éprouve aussi bien une fascination voyeuriste à espionner les ébats de jeunes couples au Prater que la haine de soi, plus particulièrement tournée contre «ce fruit poreux et rance qui marque l'extrémité de son bas-ventre ${ }^{43}$ ». Elle s'automutile. Elle implore Klemmer de la dominer et de la battre. Elle achète de jolies robes que sa mère finit invariablement par lacérer. La coquetterie lui est refusée, tout comme l'amour. Elle passe sa vie à se sentir vieillir, à se détériorer, à subir une existence de femme que rien ne vient valoriser ni sublimer, pas même le piano, car il l'assoit dans l'ombre des grands maitres. La pianiste n'est ni une compositrice, ni une virtuose ; elle ne fait qu'enseigner le piano - un aspect que reflète le titre anglais du roman: The Piano Teacher ${ }^{44}$.

Ce n'est donc pas à un avatar féminin de Benjamin Button ou d'un Struldbrugg qu'elle fait penser en définitive, mais à celui d'un Peter Schlemihl qui, au lieu de s'être départi de son ombre, se serait fait arracher sa jeunesse. Jelinek l'indique nommément : Erika se caractérise par " son absence de jeunesse ${ }^{45}$ ». À 36 ans, elle a déjà basculé, de corps et d'esprit, dans la vieillesse. Après l'épisode de la lettre, Klemmer la qualifie d'« ondine putréfiée $e^{46} »$. Ce n'est pas seulement l'insulte qu'une maîtresse névrosée a inspirée à son amant effaré par sa perversité. Erika est habitée par une force de décomposition. Elle transporte la flétrissure partout avec elle :

En allant à l'école, Erika voit partout de façon quasi obsessionnelle le dépérissement des êtres humains et des victuailles, elle ne voit que rarement croissance et prospérité. Sauf peut-être dans le parc de l'Hôtel de ville et au jardin aussi public où s'exhibent roses et tulipes charnues. Mais elles aussi se réjouissent trop tôt, car elles portent déjà en elles le temps de la flétrissure. Telles sont les pensées d'Erika. Tout la confirme dans ces pensées. Seul l'art a, selon elle, une durée de conservation supérieure ${ }^{47}$.

Le mépris dans lequel Erika tient son corps éclaire cette "durée de conservation supérieure », qui n'a en définitive rien de lénifiant: "Son corps entier n'est qu'une chambre froide où l'art se conserve ${ }^{48}$." Qui a bien pu aménager cette "chambre froide ", sinon son ogresse de mère ?...

Il n'y a peut-être aucun vocable allemand qui traduise précisément la notion d'ogre, hormis peut-être celui de Menschenfresser, mais n'empêche : la mère d'Erika a tout d'une ogresse ${ }^{49}$. Les diverses images dont use Jelinek à son propos - celles d'une " araignée ${ }^{50}$ ", d'une « vampire ${ }^{51}$ ", d'une « mère puma ${ }^{52}$ », d'une « Niobé à la retraite ${ }^{53}$ " ou d'une « vieille guêpe ${ }^{54}$ » notamment - n'ont rien de contradictoire avec le fait que Mme Kohut n'a fait qu'une bouchée de sa fille tout au long de sa vie. La dévoration annihilatrice caractérise la relation de Mme Kohut à sa fille d'une manière qui peut rappeler le lien destructeur qui unissait le Dr Paul Calmet à son fils Jean dans le roman 
de Jacques Chessex, L'Ogre (1973). Or chez Chessex, le père, tyran et rival amoureux du fils, meurt et continue d'exercer une influence mortifère sur son fils, lequel ne parvient ultimement à s'en affranchir qu'en s'enlevant la vie, autrement dit, en tuant son père en lui.

Tyrannique, assurément, Mme Kohut n'est toutefois pas la rivale amoureuse d'Erika (elle sera plutôt sa partenaire récalcitrante par un instant d'égarement érotique), pas plus qu'elle ne meurt au cours du roman (bien qu'elle se soit fait arracher des cheveux lors d'une dispute particulièrement animée). Pourtant, elle exerce une action néfaste sur sa fille qui rappelle l'emprise malsaine de «l'ogre» Calmet sur son fils Jean. Seulement, l'impuissance filiale que décrit Jelinek parait en bout de ligne plus désespérée que celle présentée dans le roman chessexien, car Erika ne parvient pas à se libérer, fût-ce en se suicidant. Le couteau qu'elle transporte dans son sac-à-main, à la fin du roman, ne lui servira qu'à se taillader gauchement l'épaule. Dans La Pianiste, le statu quo n'est pas la moins funeste des issues.

Haneke n'a pas retenu dans son film la figure de la grand-mère, qui fut autrefois complice de Mme Kohut dans son dépeçage d'Erika. Elles formaient ensemble une «brigade féminine » :

Les deux vieilles, au sexe desséché, refermé, se jettent devant tout mâle qui se présente pour l'empêcher d'arriver jusqu'à leur petite chatte. Car ce jeune animal ne doit donner prise ni à l'amour, ni au désir. Les grandes lèvres silicifiées des deux vieilles happent le vide avec des claquements secs comme les mandibules d'une lucane à l'agonie, mais aucune proie ne se laisse piéger. Aussi s'en tiennent-elles à la chair fraîche de leur fille et petite-fille qu'elles déchiquettent lentement tout en protégeant de leurs cuirasses ce sang jeune, afin que nul ne vienne l'empoisonner ${ }^{55}$.

$\mathrm{Au}$ sein de cet ordre matrilinéaire vicieux, duquel tout élément masculin est banni Jelinek ne mentionne guère le grand-père d'Erika; le père devenu fou a terminé ses jours dans un hospice pour vieillards et Klemmer ne réussit pas à s'immiscer complètement dans la vie de la pianiste -, mère et grand-mère tentent le tout pour le tout pour exercer un pouvoir féminin: "La mère autour et la grand'mère buse interdisent à l'enfant confiée à leurs soins de quitter le nid. Elles s'en prennent à SA vie qu'elles dépècent, et les voisines à $\mathrm{SA}$ réputation qu'elles mettent en pièce. Toute parcelle où frémit la moindre vie est déclarée pourrie puis sectionnée ${ }^{56}$. Voilà donc à quoi aboutit la figuration inquiétante et monstrueuse du vieillissement féminin chez Jelinek : dans ce pays pourri, où la femme n'a ni pouvoir, ni liberté, ni beauté durable en fin de compte (puisque c'est le regard masculin qui la lui prête et la lui ôte), il ne reste finalement aux femmes qu'une ressource qui soit en accord avec leur nature : la faculté de détruire.

Or «la brigade féminine », en installant cet ordre matrilinéaire, oublie qui conserve l'avantage dans ce "pays de cannibales » : l'homme. Aussi est-ce le jeune Klemmer qui va devenir « ogre ${ }^{57}$ » dans l'aire des « deux oiseaux ».

\section{Les désarrois de l'élève Klemmer}

Il ne me reste plus qu'à évoquer, en terminant, un autre point de vue qui éclaire, dans La Pianiste, la perspective du vieillir féminin: celui de Walter Klemmer, l'élève propulsé dans la position de maître. Erika jure, dans sa lettre, de lui obéir en tout : « La femme veut amollir des années de pétrification, que l'homme en profite pour la dévorer! peu lui importe. Se perdre entièrement dans cet homme, tel est son désir 
$[. . .]^{58}$.» Cependant, la dialectique maître-esclave qu'Erika appelle de ses vœux procède d'une situation qui ne se laisse guère maîtriser. Comment aimer une femme dépersonnalisée ? Comment fermer les yeux sur l'abîme de monstruosité que la lettre d'Erika a révélée, en édictant ses «instructions " quant à la façon dont Klemmer doit s'y prendre pour «l'aimer»: en la dominant, en l'humiliant, en la violentant, en l'écrasant... Klemmer a de quoi s'effaroucher, car au lieu d'une expérience susceptible de lui servir lors de ses conquêtes futures ${ }^{59}$, la séduction de son professeur de piano lui renvoie, en plein visage, l'intenable confirmation du destin mortifiant dans lequel l'homme a rivé la femme: «maintenue vivante dans des stéréotypes, la femme est une surface de projection, une image sur papier glacé, une illusion, immobile et figée $e^{60} »$. Le vieillissement des femmes agit comme un révélateur: la guerre des sexes n'est pas menée à armes égales.

Contrairement au père d'Erika, homme faible, tôt évincé, Klemmer est de la race des conquérants. Jelinek le dépeint en élève modèle et en sportif vigoureux, deux pôles de personnalité fondamentaux dans la «liaison dangereuse" qui va se nouer avec le professeur de piano. Malgré les égards pour le beau sexe dont Walter se montre spontanément capable, avec ses manières "très vieille Autriche ${ }^{61}$ ", le jeune homme demeure le produit d'une société patriarcale, qui a porté un jugement définitif sur l'être social de la femme.

Non, l'Autriche contemporaine ne fera pas de quartier à la vieille femme. Avant de nous formaliser des intrigues outrancières à la sauce Jelinek, nous devons nous rappeler à quoi sert l'acte d'écrire pour la lauréate du Nobel 2004. Dans sa conférence de réception du Prix, elle indiquait :

Écrire est-ce la faculté de se plier à la réalité, de se blottir contre ? On aimerait bien se blottir, mais que m'arrive-t-il alors? Qu'arrive-t-il à ceux qui ne connaissent pas réellement la réalité? Elle est tellement décoiffée. Pas de peigne qui pourrait la lisser. [...] La réalité est ce qui va sous les cheveux, sous les jupes et justement: arrache vers quelque chose d'autre. Comment le poète peut-il connaître la réalité, si c'est elle qui passe en lui et l'arrache, toujours vers l'écart ${ }^{62}$.

Quand Jelinek écrit la vieillesse, elle ne se plie guère à la réalité ni ne fait d'elle un objet contre lequel il serait bon de se blottir. L'image des cheveux et des jupes est parlante, puisqu'Erika va à un certain moment scalper sa mère tandis que cette dernière ne peut s'empêcher de déchirer les robes de sa fille. Il s'agit d'indices nous révélant à nous, lecteurs, que la réalité passe entre la mère et la fille.

La vie à l'écart que mènent Erika Kohut et sa mère permet à Jelinek de s'attaquer à la vieillesse comme construction sociale historiquement et culturellement marquée. Pardelà leurs deux figures, c'est une culture entière dont Jelinek dynamite les bases : « Les phénomènes qui obscurcissent la planète ne relèvent pas du pouvoir d'individus isolés. Les hommes ne sont que des organes exécutifs, qui doivent ensuite manger le gâteau ${ }^{63}$. » Le texte ne précise pas s'il s'agit du fameux « Sacher Torte »; mais à n'en pas douter, pour Jelinek, cette viennoiserie est indigeste. 


\section{NOTES}

1. Elfriede Jelinek, Das Lebewohl. 3 kl. Dramen, Berlin, Berlin Verlag, 2000, p. 56.

2. Littéralement : « celle qui souille le nid ». L'expression équivalente en français serait « celle qui crache dans la soupe ».

3. Id., La Pianiste, traduit de l'allemand par Yasmin Hoffmann et Maryvonne Litaize, Paris, Points, 2002, p. 60 .

4. Hans-Jürgen Greif, « Elfriede Jelinek : sur la brèche, sans fard, avec artifices », in : Nuit blanche, n69 (hiver 1997), p. 119.

5. Nancy Huston, «Détruire, dit-elle : Elfriede Jelinek », in : Professeurs de désespoir, Arles, Actes Sud, coll. « Babel », 2004, p. 257.

6. Ibid., p. 257-258.

7. Elfriede Jelinek, Lust, traduit de l'allemand par Maryvonne Litaize et Yasmin Hoffmann, Paris, Points, 2006, p. 212.

8. Id., La Pianiste, p. 170.

9. Id., Drames de princesses. La Jeune Fille et la Mort I-V, traduit de l'allemand par Magali Jourdan et Mathilde Sobottke, Paris, L'Arche, 2007, p. 34.

10. Cité par Pat Thane, «The 20th Century », dans : Pat Thane (dir.), A History of Old Age, Londres, Thames \& Hudson, 2005, p. 295. (« Vous croyez que c'est amusant d'être vieux? Mon satané corps tombe en ruine. » [Ma traduction])

11. Jean de la Fontaine, «Le Vieillard et les trois jeunes Hommes», Les Fables, vol. 2 : Livres VII à XII, Paris, Larousse, coll. « Classiques Larousse », 1971, p. 107.

12. Jonathan Swift, Voyages de Gulliver dans des contrées lointaines, Paris, Garnier Frères, 1856, p. 316-317.

13. Elfriede Jelinek, La Pianiste, p. 168.

14. Ibid., p. 69.

15. «En outre, la valeur d'une femme décroît fortement à mesure qu'elle avance en âge et progresse en intelligence. » (Ibid., p. 148.)

16. Ibid., p. 180.

17. Id., Méfions-nous de la nature sauvage, traduit de l'allemand par Yasmin Hoffmann et Maryvonne Litaize, Paris, Points, 2011, p. 11.

18. David Cravitt, The New Old, Toronto, ECW Press, 2008, p. 2. ("Ce que font les boomers consiste, tout simplement, à détruire notre conception générale du vieillissement. Les boomers sont, en effet, en train de dé-vieillir. » [Ma traduction])

19. Elfriede Jelinek, Méfions-nous de la nature sauvage, p. 12.

20. Ibid., p. 13.

21. Voir Vanessa Besand, "L'œuvre romanesque d'Elfriede Jelinek: une esthétique de la pop culture? », Trans-, n9 (hiver 2010), 9 p. <http://trans.univ-paris3.fr/> Consulté le 15 janvier 2012.

22. Elfriede Jelinek, Totenauberg, traduit de l'allemand par Yasmin Hoffmann et Maryvonne Litaize, Paris, Points, 2011, p. 12.

23. Id., Méfions-nous de la nature sauvage, p. 63.

24. Id., Totenauberg, p. 25.

25. Ibid., p. 13.

26. Id., La Pianiste, p. 137.

27. Id. dans Hans-Jürgen Greif, op. cit., p. 119.

28. Id., La Pianiste, p. 9.

29. Ibid., p. 249. 
30. Ibid., p. 57.

31. Ibid., p. 19.

32. Ibid., p. 6.

33. Ibid., p. 168.

34. Ibid., p. 56.

35. Ibid., p. 170.

36. Francis Scott Fitzgerald, "The Curious Case of Benjamin Button », in Tales of the Jazz Age, New York, Charles Scribner's Sons, 1922, p. 192-224.

37. Elfriede Jelinek, La Pianiste, p. 101.

38. Nicole Bary, «Présentation », in Elfriede Jelinek, Lust, p. iv.

39. Loc. cit.

40. Elfriede Jelinek, La Pianiste, p. 174.

41. Ibid., p. 19.

42. Ibid., p. 39.

43. Ibid., p. 175.

44. Id., The Piano Teacher, traduit en anglais par Joachim Neugroschel, New York, Weidenfeld \& Nicolson, 1988.

45. Ibid., p. 178.

46. Ibid., p. 194.

47. Ibid., p. 80.

48. Ibid., p. 17 .

49. Sur le mythe de l'ogre dans la littérature, voir les travaux de Jonathan F. Krell, en particulier l'ouvrage The Ogre's Progress. Images of the Ogre in Modern and Contemporary French Fiction, Newark, University of Delaware Press, 2009.

50. Elfriede Jelinek, La Pianiste, p. 31.

51. Ibid., p. 34.

52. Ibid., p. 135.

53. Ibid., p. 138.

54. Ibid., p. 183.

55. Ibid., p. 28.

56. Ibid., p. 29.

57. Ibid., p. 184.

58. Ibid., p. 183.

59. «Le commerce d'une femme nettement plus âgée - avec laquelle les égards sont devenus inutiles - lui permettra de découvrir comment s'y prendre avec les jeunes filles qui ne se laissent pas faire si facilement. » (Ibid., p. 56.)

60. Magali Jourdan et Mathilde Sobottke, dans Id., Drames de princesses, p. 3.

61. Elfriede Jelinek, La Pianiste, p. 61.

62. Id., "Conférence Nobel de littérature 2004. À l'écart », traduit de l'allemand par Louis-Charles Sirjacq, Nobelprize.org <http://www.nobelprize.org/nobel_prizes/literature/laureates/2004/ jelinek-lecture-f.html> Consulté le 15 janvier 2012.

63. Id., Totenauberg, p. 29. 


\section{RÉSUMÉS}

Cet article se propose d'examiner la manière dont la romancière et dramaturge autrichienne Elfriede Jelinek (née en 1946) approche la question du vieillissement des femmes dans une société qu'elle juge patriarcale et aliénante. En nous basant principalement sur son roman le plus connu, La Pianiste (1983), nous verrons que le choix d'approcher les deux protagonistes féminins, Erika Kohut et sa mère, sous l'angle de leur âge avancé, est révélateur du rôle que Jelinek entend remplir comme écrivain, à savoir se livrer à une violente critique sociale et dénoncer les esclavages modernes par le truchement du sarcasme et de l'outrance. Nous procédons ici en deux étapes : nous faisons d'abord ressortir la dynamique du vieillissement depuis l'époque où Jelinek a entamé sa carrière littéraire. Ensuite, nous examinons tour à tour la figure d'Erika, cette «vieille » femme de 36 ans, puis celle de Mme Kohut, sa mère âgée dont le caractère possessif dénote une nature d'ogresse. Vieillir, dans ce contexte, ne fait qu'aggraver une condition déjà intenable pour la femme.

This paper aims to discuss how the Austrian novelist and playwright Elfriede Jelinek (born in 1946) addresses the issue of aging for women in a society she regards as patriarchal and alienating. Our analysis is principally based on her most praised novel, The Piano Teacher (1983). In her depiction of the two female protagonists, Erika Kohut and her mother, Jelinek focuses on their old age. By doing so, she reasserts what she considers to be her role as a writer: to fiercely criticize modern society and to denounce its slaveries through sarcasm and outrageousness. This paper applies a two-step approach: first, we bring out the dynamics of aging since the beginning of Jelinek's literary career. Then, we successively examine Erika (the "elderly" 36 year-old woman) and Mrs. Kohut, her aged mother whose possessive nature indicates an ogress status. Aging, in this context, only worsens a situation that is already unbearable for women.

In diesem Artikel wird gezeigt, auf welche Weise die österreichische Romanschriftstellerin und Theaterautorin Elfriede Jelinek (geb. 1946) den Altersprozess der Frau innerhalb einer Gesellschaft darstellt, die sie als patriarchalisch und entfremdend beurteilt. Wir stützen uns vor allem auf ihren bekanntesten Roman, Die Pianistin (1983). In diesem Text wird die Rolle deutlich, die Jelinek den beiden weiblichen Hauptfiguren - Erika Kohut und deren Mutter - ihres Alters wegen zuweist. Zugleich profiliert sie die Funktion der Schriftstellerin, die für sie eine scharfe soziale Kritikerin sein soll, die sarkastisch und stark überspitzt die Formen moderner Sklaverei anprangern muss. Wir beschränken uns hier auf zwei wesentliche Punkte: Zum einen zeigen wir die Dynamik des Alterns, die seit Beginn der Tätigkeit Jelineks als Schriftstellerin in ihrem Werk vorhanden ist. Zum anderen untersuchen wir die Figur Erikas, dieser «alten» 36-jährigen Frau sowie die ihrer betagten Mutter, deren besitzergreifender Charakter Wesenszüge der «Menschenfresserin» aufweist. In diesem Kontext bedeutet Altern das Verschärfen einer Situation, die für die Frau in jedem Fall unerträglich ist.

\section{INDEX}

Mots-clés : féminité, roman, vieillesse

oeuvrecitee Die Klavierspielerin, Lust, Winterreise, Wir sind lockvögel baby! 
AUTEUR

\section{PATRICK BERGERON}

Université du Nouveau-Brunswick 